\title{
Review Article \\ On the Development of Focused Ultrasound Liquid Atomizers
}

\author{
Ahmed M. Al-Jumaily and Ata Meshkinzar \\ Institute of Biomedical Technologies, Auckland University of Technology, Private Bag 92006, Auckland 1142, New Zealand \\ Correspondence should be addressed to Ahmed M. Al-Jumaily; ahmed.al-jumaily@aut.ac.nz
}

Received 15 September 2016; Accepted 22 January 2017; Published 6 February 2017

Academic Editor: Marc Asselineau

Copyright (C) 2017 Ahmed M. Al-Jumaily and Ata Meshkinzar. This is an open access article distributed under the Creative Commons Attribution License, which permits unrestricted use, distribution, and reproduction in any medium, provided the original work is properly cited.

This paper reviews the evolution of focused ultrasonic transducers of various kinds for fluid atomization and vaporization. Ultrasonic transducers used for atomization purposes in biomedical, pharmaceutical, or industrial applications, such as surface acoustic wave (SAW) transducers, array of micromachined nozzles, and Fourier horn micromachined nozzles with or without a central channel, are all presented and compared. For simplicity of manufacturing and low cost, we focus on plates and curved and corrugated structures for biomedical humidification.

\section{Introduction}

Ultrasonic transducers have been receiving a great deal of attention during the past few decades because of their wide variety of applications as sensors or actuators in flaw detection, thickness gaging, materials research and medical diagnostics, and sonar. In many biomedical, pharmaceutical, and industrial applications, ultrasonic transducers can be used as atomizers. Humidifiers, micro/nanoelectronics, nanoparticles synthesis, spray coating, drug delivery, drug preparation for inhalation, and others are among the most common applications of atomization for which ultrasonic transducers can play an important role and can be extremely beneficial. Droplets $<10 \mu \mathrm{m}$ in diameter are highly desirable for various medical purposes. For pulmonary microcirculation-related applications, the droplets must be smaller than $7 \mu \mathrm{m}$ in diameter to safely pass through the microvessels of the lung without causing obstruction [1]. For delivering drugs to the respiratory system, droplets of 3 to $5 \mu \mathrm{m}$ are ideal depending on the disease and its site [2]. For delivery of medications to the alveolar capillary bed, $1-3 \mu \mathrm{m}$ droplets (optimal at $2 \mu \mathrm{m}$ ) are ideal $[2,3]$. However, delivery for humidification, such as in lung therapy, requires humidified air where water particles are much smaller than $1 \mu \mathrm{m}$.

Inhalation is an attractive route for noninvasive delivery of drugs [4-7] especially peptides and proteins that are easily broken down by enzymes in the stomach when taken orally $[4,6]$. The wide variety of potential applications of ultrasonic transducers justifies their in-depth analysis and investigation. The shape and geometry of the transducer as well as its modes of vibration play the most significant roles in its performance. Various types of transducer geometries and shapes such as flat, curved and corrugated plates, and cylindrical and spherical shells have been implemented for ultrasound generation. The driving mechanism for this generation is the vibration characteristics of the transducer. To quantify the power and strength of the signal, it is essential to understand those characteristics. Analytical, numerical, and experimental investigations have been conducted on various transducers and their vibration characteristics were examined in the existing literature. Different approaches were followed by authors and various designs were devised for the transducers making them applicable to suit specific requirements.

Atomization is an important application of ultrasonic transducers, in particular in biomedical applications. Therefore, some researchers have focused on the mechanism of the atomization itself which is definitely of great help and importance to further improve the performance of the transducers. Two mechanisms were considered by various researchers for atomization, breaking up of capillary waves at the liquid surface and cavitation. However, further research showed that a combination of these two mechanisms is the source of atomization $[8,9]$. Most of these studies focus on 
the performance of such devices for atomization rather than evaporation. However, some investigations have dealt with methods to evaporate water droplets. These methods include resonant excitation and disintegration in air, acoustic squeezing and disintegration, and finally solid surface excitation. Droplets of water can be acoustically levitated by means of an acoustic levitator resulting in the oscillation and disintegration of the water droplets [10, 11]. A similar approach was attempted to acoustically squeeze a droplet to the point where it flattens out and self-disintegrates $[12,13]$. The interaction of water droplets with solid surfaces vibrating at ultrasonic frequencies was also investigated [14-16]. Current commercial biomedical devices (Misty-Neb, Aero Eclipse, Omron, Pari eFlow, and Philips I-neb) producing droplets or aerosols by compressed air, a vibrating piezoelectric plate together with a metallic mesh or a vibrating mesh, suffer from broad droplet size distributions and low throughput, which makes it difficult to deliver sufficient dosages of drugs precisely and rapidly to the targeted sites $[17,18]$. Furthermore, the eFlow and I-neb which utilize vibrating mesh technology [19] and are considered the most advanced commercial devices suffer from clogging of the mesh orifices [20]. Heating can also be used to atomize and produce droplets. However, ultrasonic atomization provides significant energy and space savings compared to conventional heated humidifiers [21]. Therefore, further improvement of available devices or devising new compact atomizers capable of producing water vapor rather than water droplets is of practical importance, in particular in medical therapy devices such as those used for lung therapy. It is worth noting that an inseparable part to further improve existing vaporizing devices and technologies for atomization is to thoroughly investigate and flourish the vibration characteristics of the transducers. Consequently, an important brief but essential review will also be presented in Section 2 regarding flat plate and shell type transducers followed by another section on atomization methods.

Since the information on the area is widespread and the research field is of great significance, the authors decided to merge many of such topics into a single document which will serve as a helpful resource and guideline for many of those working in the field.

\section{Ultrasound Generation}

2.1. Vibrating Plate Radiators. Achieving ultrasonic frequencies for gases utilizing conventional ultrasonic transducers has been difficult with low efficiency. Further, electromagnetic, magnetostrictive, and piezoelectric ultrasound sources seem incapable of generating high power ultrasounds with good efficiency and directivity as they cannot undergo large longitudinal vibration amplitude. The problems lying behind ultrasound generation in gases are attributed to low specific acoustic impedance and high absorption of the medium. Therefore, in order to have an efficient energy transmission, good impedance matching between the transducer and gas medium, large amplitudes of vibration and highly directional radiation are needed [22]. Vibrating plates can be used as ultrasonic radiators in fluids; however, the directivity of the generated acoustic waves is a matter of great importance.
The radiations from different parts of a simple flat plate with constant thickness are in counter phase leading to phase cancellation and, therefore, poor directivity. On the contrary, if one considers a plate with some steps on the surface raised half a wavelength of the radiated sound, the radiations become in-phase and directivity increases. Several experimental and analytical approaches have been attempted to determine the improvements achieved in delivering stronger ultrasound using various plate geometries and shapes. Circular aluminum plate transducers with steps were designed and their vibration characteristics were investigated experimentally and analytically. Using uniform plate solution and the energy method, an analytical solution was obtained for the frequency equation by matching boundary conditions at the step interface and free edge condition at the external boundaries [23, 24]. Good agreement was reported between the experimental and the analytical approaches and better directivity. The structure of the transducer and the radiating plate consists of three concentric copper rings with small holes to spray water jets to the nodal circles to cool them. High intensity ultrasound was reported to have been generated in gases with high efficiency (approx. 80\%) and directivity. Analytical vibration analysis of stepped plates without any restriction on the number of steps or on the order of the vibration mode, unlike the earlier works in the literature which were restricted to one step and/or first vibration mode, was conducted by San Emeterio et al. [25]. The analytical results agreed well with those of experiments in thick areas of the plate, while discrepancies were observed in thin regions. Therefore, in their analytical model for the deformation function, they proposed a correction factor taking the effect of the base plate and step thicknesses ratio into account and obtained good agreement between their analytical and experimental results. The influence of Poisson's ratio on the natural frequencies of stepped-thickness circular plate has been also dealt with [26]. In that paper, Al-Jumaily and Jameel determined the natural frequencies of the simply supported and clamped stepped plates using classical plate solutions with exact continuity conditions at the step. They concluded that larger Poisson's ratio indicates stiffer plates and larger natural frequencies. Also as the step size increases, the natural frequencies are expected to be larger. They also stated that Poisson's ratio should not be disregarded in the continuity equation in particular for the fundamental frequency.

To have directional underwater radiation, two design methods were introduced by Montero de Espinosa and Gallego-Juárez [27]. In one method, they implemented helical waveguides and in the other one, a delaying liquid was used with the same acoustic impedance as water, but half of the sound velocity as in water. This way, they made inphase the regions of the plate which had been previously radiating in counter phase resulting in good directivity for the frequency range of $20-27 \mathrm{kHz}$.

High power generators are needed to make use of sonic and ultrasonic energy in industrial applications. However, their use has been restricted by the inadequacy of such generators. To this aim, Gallego-Juárez et al. worked on the design and development of macrosonic generators for industrial applications taking into account good impedance matching 
between the transducer and the medium for efficient transmission of energy, high directional or focused radiation for energy concentration and high amplitude of the operating mode for intense acoustic radiation, fatigue failure of the material, the distribution and location of the stresses, and the isolation of the operating vibration mode from other close nontuned modes [28]. Finally, double-stepped rectangular titanium plates were devised achieving high power and good performance in air $(7.6 \mathrm{kHz}$ with the applied power of around $2 \mathrm{~kW}$ had the efficiency of $67 \%$ and dimensions of $1.8 \times$ $0.9 \mathrm{~m}^{2}$ ). Gallego-Juárez et al. have conducted further research to come up with a solution for large industrial applications of high intensity transducers for air where numerous practical problems are encountered due to the required high-order vibration modes [29]. Another solution was proposed for low sonic applications where stepped-plate transducers seem unsuitable as the height of the steps, which has to be half a wavelength of the radiation, becomes too high and it makes the transducer construction impractical. For the industrial case, they used an array of five circular stepped-plate transducers (each plate $48 \mathrm{~cm}$ diameter, $21 \mathrm{kHz}$, applied power $350 \mathrm{~W}$ and $75 \%$ efficiency). For the case of low frequency applications in air, a plate transducer with reflectors was used making the radiation in-phase leading to high directivity.

The above literature reveals that for many applications from low sonic transducers to ultrasonic radiation in gas or fluid medium including underwater sonar, manipulations on the geometry of flat plates of different kinds, that is, rectangular or circular, led into a solution suited for that specific application. The common theme for all the cases is that the radiation was made focused and in-phase by geometrical manipulations. Therefore, similar ideas can be employed for smaller biomedical humidifier applications.

2.2. Shell Transducers. In order to gain a better understanding of an ultrasonic transducer, it is essential to investigate its vibration characteristics for various design configurations. In order to increase the focus and directivity, curved structures can be a suitable candidate. Therefore, for airborne ultrasonic ranging measurement, a partially cylindrical (curved) PVDF transducer with silver electrode on both sides was reported to effectively couple ultrasound into the air and generate strong sound pressure [30]. The transducer was observed to have two resonances as length extensional mode and flexural bending mode. Transient surface displacement measurements revealed that vibration peaks were in-phase for the length extensional mode and out of phase for the flexural bending mode generating a stronger ultrasound wave for the length extensional mode. The resonance frequencies and vibration amplitudes of the two modes strongly depend on the structure parameters as well as the material properties. Controlling the thickness of electrode is important as well since it affects the resonance characteristic of the transducer. The resonance frequencies of the two modes should be separated as far as possible from each other to minimize the influence of bending vibration. The effect of variable curvature on the transducer performance was not known. Therefore, Toda and Tosima investigated the vibration modes of a curved, clamped, piezoelectric multilayer film with uniform and nonuniform film curvature and it was concluded that the nonuniform curvature generates much higher output pressure in air than a uniform one [31]. They did not mention the highest achievable sound pressure level in decibel using their proposed piezoelectric multilayer film. However, according to Figures 9 and 10 in their work where the output acoustic pressure is depicted for various frequencies, the highest sound pressure is approximately $33 \mathrm{~Pa}$ which corresponds to $124 \mathrm{~dB}$ using the unanimous formula of sound pressure level $(\mathrm{dB})=20 \log p+94$, where $p$ is the sound pressure in Pascal.

Since the curvature proved to be influential in increasing the focus and improving the directivity, the next attempt was to implement a row of curvatures placed next to each other forming a corrugated structure. A corrugated PVDF film air transducer was scrutinized to achieve a high power output and a sharp beam angle [32]. The vibration phase of convex section is shifted 180 degrees from the concave section. These waves add constructively to form a strong acoustic beam when the corrugation height is a little larger than one-half of the wavelength since the vibration is distributed over all of the points on the film. The vibration characteristics of axially symmetrical annular corrugated shell piezoelectric transducers were also analyzed [33-35]; however, the motion for such a transducer with fixed edges was reported to be complicated and therefore a global analysis was necessary in order to increase the efficiency of such transducers in operating modes. Blum et al. devised and investigated a twodimensional, air-coupled array for the noncontact generation of ultrasound [36]. The overall design objective was to position 20 electrostatic transducers in such a way that the signal amplitude at the focal line of the array gets maximized. Then, to identify the most critical parameters affecting the predicted behavior of the array, a sensitivity study was performed and it was concluded that changes in the spatial position of the transmitting transducers have a noticeable effect on the signal at the focal line. The sound pressure level of $142.70 \mathrm{~dB}$ SPL was achieved in air. Measurements showed that the amplitude of the ultrasonic waves generated with the air-coupled array is in the order of five times lower than that of a piezoelectric contact transducer.

In therapy devices, in particular those used for lungs, we always look for pure water vapor, not droplets, in the air. In spite of the fact that all of the above devices have shown some ultrasound improvement in directivity and power generation, nevertheless none of them are suited to deliver this objective. However, with the aim of producing an ultrasonic field for drying foodstuff, an aluminum cylindrical chamber was designed which was driven by a piezoelectric transducer [37]. A high intensity acoustic field was obtained inside the tube ( $155 \mathrm{~dB}$ of SPL) with relatively low industrial power applied $(75 \mathrm{~W})$. Although this is the only paper which shows a complete water evaporation process, nonetheless it requires very high power; it is large in size and not suited for biomedical humidification such as in lung therapy. In fact, for therapy humidification in particular for the lung, a smaller size transducer with less power and a shape which fits in the air delivery line would be impeccable.

This section clarifies the effect of various shell type geometries on the directivity and intensity of the acoustic 
field generated. Although none of the references dealt with the atomization or direct biomedical application, the results seem promising since geometrical manipulations on shell type structures created focused radiation. Thus, such an idea can be converted and well suited to the specific requirements of any biomedical application as atomization, humidification, drug delivery, and others.

\section{Ultrasonic Transducers as Atomizers}

Ultrasonic transducers have been extensively used for droplet generation and atomization and, as already mentioned, their application varies from ultrasonic humidification, micro/nanoelectronics, nanoparticles synthesis, spray coating to drug delivery and drug preparation for inhalation. In this section, their application for atomization purposes is summarized. In the end, a comparison among the available existing methods will be performed in the form of a table to discuss and illustrate the advantages and disadvantages of each method.

Lass et al. presented a paper on the current vibrating membrane nebulizer technology for drug delivery and dealt with current devices in the market which can be of interest [19]. Compressed air and vibrating piezoelectric plates together with a metallic mesh or a vibrating mesh are common technologies for producing aerosols or droplets in commercial devices (Misty-Neb, AeroEclipse, Omron, Pari eFlow, and Philips I-neb). A disadvantage is their broad droplet size distributions and low throughput making it difficult to deliver sufficient dosages of drugs precisely and rapidly to the targeted sites $[17,18]$. In addition, blockage of mesh orifices in the vibrating mesh membrane of eFlow and I-neb which are considered to be the most advanced commercial devices is a pitfall [20]. Recent in vivo studies have indicated that in both adults and children, when inhaling typical aerosols from current commercial devices, the upper airways [48], ventilator, and endotracheal tubes are significant barriers to lung deposition [49]. As a direct result of poly disperse droplet size distributions, drugs are delivered to nontargeted sites, resulting in harmful side effects in the pharynx and losses in the ventilator/endotracheal tubes. Therefore, investigation and development of ultrasonic transducers as atomizers in order to improve their performance are a matter of great importance.

In 1988, Elrod et al. worked on droplet formation using tone bursts of focused acoustic energy [50]. In order to generate a spherically converging acoustic beam, a focusing element (acoustic microscope lens) was used without any nozzle. The liquid surface was adjusted to be at the focal plane, where the beam was concentrated. Droplets of 300 to $5 \mu \mathrm{m}$ were generated within the frequency range of 5 to $300 \mathrm{MHz}$.

3.1. Vibrating Plate Atomizers. Vibrating plate transducers are commonly being used for humidifiers and other applications since they possess the best combination of performing specifications compared to other types of devices. In these atomizers, an electrical signal is converted to mechanical oscillation using a piezoelectric material immersed in a reservoir of water. The ultrasonic waves created by the mechanical vibration of the plate are directed towards the water surface creating a mist of water droplets. These atomizers are normally available in a high frequency range of 1.65$3 \mathrm{MHz}$ which are capable of producing droplets within the range of $1-5 \mu \mathrm{m}$ in diameter using 2 up to $30 \mathrm{~W}$ of power. The flow rate varies within the range of $5-400 \mathrm{~mL} / \mathrm{h}$. They are in contact with water and the water level on their top affects their performance and should be taken into account for the optimum performance of the device. They are available in various plate area dimensions and thicknesses. Examples of such atomizers can be referred to in references [44-47]. Their flow rate also depends on many external factors such as input power, droplet size, frequency and liquid quality, temperature, and level (depth). The good point about them is that they can produce various sizes of droplets and flow rates which makes them suitable for some applications. As already mentioned, flat plates do not produce focused ultrasonic waves. Therefore, curved transducers have been used in some of the aforementioned atomizers providing a little bit of better focus and performance compared to the flat ones as evident in [44-47]. However, depending on the application, a combination of high flow rate, small droplet size, and low power demand may be required which has not been achieved yet. Therefore, seeking alternative ways to create more focused ultrasonic waves seems essential and can lead to the better performance of the device. Going to higher frequencies to obtain more focused waves resulting in better performance of the device can lead to overheating and depolarization of the material [51] and also fatigue failure [28]. Therefore, it is a restricting factor and alternative ways should be sought. One alternative is using stepped-thickness plates. Although, they have been investigated and proven to be useful and practical as discussed elaborately in Section 2.1, to the authors' knowledge they have not been implemented in the current vibrating plate atomizers available in the market. To overcome overheating, Lozano et al. designed an electronic system to excite piezo-ceramic disks for ultrasonic atomization [51]. It was designed based on a DC-AC converter with $\mathrm{H}$-bridge topology switching ZVS (zero voltage switching) mode optimized for R-L-C type loads. In order to reduce ceramic overheating, the system operated in burst mode sending a predetermined number of pulses. There was no sign of atomization for voltages below $10 \mathrm{~V}$. Excitation bursts of 80,000 pulses at a repetition rate of $1 \mathrm{kHz}$ were applied to the transducers. No atomization was initiated at bursts formed by less than 10,000 pulses and only a small proportion of the power consumption led to mechanical vibration, while a large part was dissipated as heat. The resulting droplet size distribution showed two main peaks at 3.5 and $5.5 \mu \mathrm{m}$. Droplet diameter was observed to be independent of the excitation amplitude (voltage), while the atomization rate increased with voltage.

3.2. Surface Acoustic Wave Atomizers. Kurosawa et al. proposed a novel way to produce dry fog using a surface acoustic wave (SAW) transducer of $\mathrm{LiNbO}_{3}$ piezoelectric substrate as in Figure $1[38,39]$. The atomizer consists of a vibrator which has an interdigital aluminum transducer (IDT) consisting of 20 pairs of electrodes supplied with RF (radio frequency) power amplifier at $48 \mathrm{MHz}$ frequency. The surface wave called 


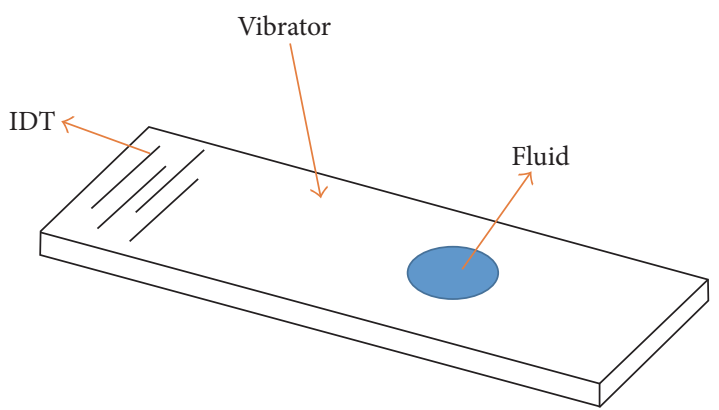

Figure 1: Surface acoustic wave atomizer.

capillary wave generated by the radiation of acoustic wave from the SAW device surface was capable of atomization when the liquid layer on the surface was half a millimeter or less. The atomizing mechanism was reported not to be vapor but spray from crests of the surface wave in a fluid. The mean diameter of the mist was about $5 \mu \mathrm{m}$. The atomizing rate was $170 \mu \mathrm{L} / \mathrm{min}$ at $2.3 \mathrm{~W}$ input power $(36 \mathrm{~V})$. Applying $100 \mathrm{MHz}$ was reported to be capable of reducing the size of the device to an extent suiting it for medical applications such as spraying a liquid medicine to the diseased target directly with the atomizer on an endoscope.

A low driving power SAW atomizer was developed consisting of a unidirectional interdigital transducer, a horn, and a waveguide fabricated on $\mathrm{LiNbO}_{3}$ substrate to increase the focus [40]. For continuous atomization of water to get droplets of $1.5 \mu \mathrm{m}$ and $40 \mu \mathrm{L} / \mathrm{min}$ flow rate, $1 \mathrm{~W}$ of driving power was required at a frequency of about $78 \mathrm{MHz}$. Low driving power and small droplets make the atomizer attractive for practical applications in chemistry, biology, and medicine.

Ju et al. constructed and tested high frequency SAW atomizers, with resonance frequencies in the range 50$95 \mathrm{MHz}$ [52]. A liquid sample was charged by a high-voltage (about $5 \mathrm{kV}$ ) conductive wire located just above the SAW device. Mean diameters of original droplets formed by atomization were estimated to be $5.7(50 \mathrm{MHz}), 4.4(75 \mathrm{MHz})$, and $2.7 \mu \mathrm{m}(95 \mathrm{MHz})$, respectively. Based on the test, the minimum power required for atomization was approximately $4 \mathrm{~W}$ $(50 \mathrm{MHz}), 11 \mathrm{~W}(75 \mathrm{MHz})$, and $24 \mathrm{~W}(95 \mathrm{MHz})$ and atomization speed was $0.06 \mu \mathrm{L} / \mathrm{s}(50 \mathrm{MHz}), 0.04 \mu \mathrm{L} / \mathrm{s}(75 \mathrm{MHz})$, and $0.01 \mu \mathrm{L} / \mathrm{s}(95 \mathrm{MHz})$. The high-voltage needed made the use of such atomizer restricted.

3.3. Ultrasonic Horns. High ultimate particle velocity, low acoustic loss, and high thermal conductivity make silicon ideal for high amplitude ultrasonic applications superior to titanium alloys [53]. Therefore, high amplitude ultrasound was generated by a micromachined silicon resonant transducer to atomize liquids. Lead-zirconate-titanate (PZT) plates were used to drive the needle-shaped device which was made by bonding two silicon horns. Water was atomized continuously at a flow rate of $2.4 \mathrm{~mL} / \mathrm{min}$ into a mist with mean drop size of $25 \mu \mathrm{m}$ at $72 \mathrm{kHz}$ resonance frequency.
In order to develop a portable device for internal body therapeutic applications, Li et al. devised a $421 \mathrm{kHz}$ miniaturized piezoelectric ultrasonic transducer of one-wavelength design based on the longitudinal vibration mode with a stepped horn to focus the energy [54]. The horn effect was to magnify the vibration amplitude noticeably. The PZT material selected was PZT8 which is widely used for high power applications and brass electrodes were used. Finite element analysis and equivalent circuit models were applied for theoretical analysis. The device was immersed by a quarter of the wavelength and the directivity pattern was omnidirectional within the range from 30 to 150 degrees. The potential applications of the transducers are sonodynamic therapy, drug delivery, and microfluidic pumping. It was also noted that the efficiency decreases as the voltage increases (input power increases more rapidly compared to output power; however, the intensity increases monotonically with increasing voltage). The possible reason was mentioned to be increasing losses at the high-voltage range.

Tsai and others investigated, built, and tested microelectro-mechanical system- (MEMS-) based miniaturized silicon ultrasonic droplet generators of a new and simple nozzle architecture with multiple-Fourier horns in resonance with and without a central channel $[17,18,43,55,56]$. A Schematic is depicted in Figure 2. The silicon resonator is made of a multiple-Fourier horn section where each horn is half-wavelength long with a longitudinal vibration amplitude magnification of 2 . The drive section includes a piezoelectric plate transducer bonded to the rectangular silicon base using silver paste. When PZT transducer plates are excited at the nozzle resonance frequency, a standing acoustic wave is created through the nozzle with maximum longitudinal vibration (displacement) at the nozzle tip. As a result of the vibration, standing capillary waves are formed on the free surface of the liquid as it issues from the nozzle tip. Atomization occurs by the breakup of these standing capillary waves. It is worth noting that when the ultrasonic drive frequency deviated from the nozzle resonance frequency by more than $1.5 \mathrm{kHz}$, a large liquid drop with a diameter greater than the tip width was reported to be formed at the nozzle tip without any atomization taking place.

Tsai et al. produced monodisperse ethanol droplets of $2.4 \mu \mathrm{m}$ and water droplets of $4.5 \mu \mathrm{m}$ in diameter in ultrasonic atomization using 1.5 and $1.0 \mathrm{MHz}$ MEMS-based silicon nozzles, respectively, each consisting of 3 Fourier horns in resonance (with a central channel), required electrical drive power as low as $0.25 \mathrm{~W}$ and supply flow rates as high as $350 \mu \mathrm{L} / \mathrm{min}(21 \mathrm{~mL} / \mathrm{h})$ [43]. At the resonance frequency, the measured longitudinal vibration amplitude at the nozzle tip increases as the number of Fourier horns $(n)$ increases in good agreement with the theoretical values of $2^{n}$. Using this design allows for very high vibration amplitude gain at the nozzle tip resulting in no reduction in the tip cross-sectional area for contact of liquid to be atomized. This leads to a noticeable reduction in the electric drive power which in turn decreases the possibility of transducer failure during atomization. They also extended their work to externally liquid fed ultrasonic nozzles without a central channel [17]. Droplets with a diameter range $2.2-4.6 \mu \mathrm{m}$ for alcohol (2.9-4.6 for 


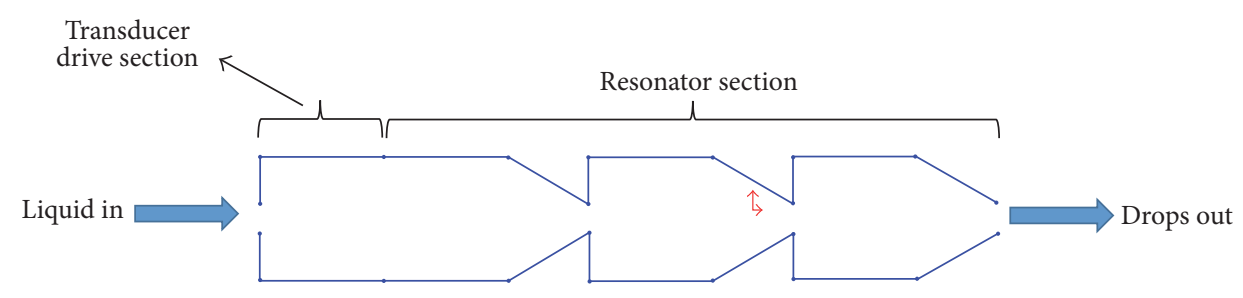

FIGURE 2: Schematic of a multiple-Fourier horn ultrasonic nozzle.

water) were produced at high throughput of $420 \mu \mathrm{L} / \mathrm{min}$ and very low electrical drive power of $80 \mathrm{~mW}$. The electrical loss for the lossy PZT transducer was reported to increase with its thickness. Moreover, the nozzles with a central channel require higher drive power for atomization which is due to the fact that they need a pair of PZT transducers. The range of drive power measured was extremely lower than that required in conventional ultrasonic atomization using $\mathrm{MHz}$ disk transducers which was attributable to the new nozzle architecture requiring only a single basic nozzle without a central channel and, thus, a single PZT transducer for activation. The optimum number of Fourier horns was found to be 3 or 4 with electrical drive power significantly below $0.1 \mathrm{~W}$ at throughput of $100 \mu \mathrm{L} / \mathrm{min}$, while the 2-Fourier horn nozzle required a drive power of $0.6 \mathrm{~W}$ to initiate atomization. In contrast, no atomization could be initiated with the 1-Fourier horn nozzle even at a drive power as high as $0.8 \mathrm{~W}$. Batch fabrication of nozzles with similar or different design specifications on a common silicon wafer reduces the costs according to the authors. Utilizing the same approach as above, Tsai et al. designed and built a small nozzle requiring low drive power with a pocket-size ultrasonic nebulizer [18]. Various pulmonary drugs were nebulized utilizing the pocket-size unit with different aerosol sizes and output rates. The obtained results demonstrated the capability of the silicon-based MHz MFHUNs (multiple-Fourier horn ultrasonic nozzle) for production of monodisperse droplets of desirable size ( 2 to $5 \mu \mathrm{m}$ ) and moderate output (up to $0.2 \mathrm{~mL} / \mathrm{min}$ ) at low electrical drive power (sub-Watt). At the typical output rate of $0.15 \mathrm{~mL} / \mathrm{min}$ with $3.5 \mu \mathrm{m}$ diameter droplets, the required electrical drive power was $0.27 \mathrm{~W}$. It was reported that a higher output rate can be accomplished readily by using an array of identical ultrasonic nozzles. With $2 \mathrm{MHz}$ of frequency, droplets of $3.1 \mu \mathrm{m}$ were produced and the output was $350 \mu \mathrm{L} / \mathrm{min}$.

3.4. Active and Passive Mesh Membranes. Mesh membranes have also been used for atomization purposes. In some of the cases, the mesh membrane vibrates itself and expels the liquid out of the mesh orifices, whereas in some other cases the liquid is between the mesh membrane and another membrane which is vibrating [19]. The first is called active and the latter is called passive mesh membrane. A new type of piezoelectric array microjet was introduced for drug delivery consisting of a fluid chamber, which was formed by a piezoelectric actuator bonded to a silicon chip with nozzles (passive mesh membrane) [41, 42]. Droplets of 5 to $10 \mu \mathrm{m}$ diameter resulted using piezoelectric transducers operating at around $36 \mathrm{kHz}$ to actuate arrays of $5 \mu \mathrm{m}$ diameter micromachined nozzles. Following the same approach as in Figure 3, Meacham focused on a piezoelectrically driven, micromachined atomizer concept that utilizes fluid cavity resonances in the $0.5-5 \mathrm{MHz}$ range combined with acoustic wave focusing for droplet generation or jet ejection [57]. This simple technique capable of producing droplets of sub$5 \mu \mathrm{m}$ diameter (D50 was $4.9 \mu \mathrm{m}$ ) had low-temperature and low power operation. It also had low cost fabrication with the capacity to scale throughput up or down by using an array. It was reported that when the piezoelectric transducer is driven at the fundamental cavity (fluid reservoir) resonance frequency or any of the higher cavity modes, a standing acoustic wave develops and constructive interference in the pyramidal nozzle focuses the wave so that the peak pressure gradient occurs near the tip of the nozzle and causes the liquid to be ejected through the nozzles as droplets. The geometry of the chamber can be readily modified to increase or decrease the driving frequency of operation (for example by increasing or decreasing the height of the cavity) according to the desired specifications. Results were presented for various affecting parameters with various orifice diameters at various AC voltages (10, 30, and $36 \mathrm{~V})$ having different input power. Experiments performed at different resonance frequencies revealed that the diameter of the ejected droplets decreases with increasing frequency. Doubling the thickness of the piezoelectric transducer reduces the longitudinal resonance of the transducer to half of the original frequency. The power transfer from the piezoelectric transducer to the fluid was more efficient at lower frequencies near the fundamental cavity resonant mode.

Jeng et al. devised a PZT-driven atomizer consisting of a flexible membrane and a micromachined trumpet-shaped nozzle array [58]. The atomizer employed a PZT bimorph plate attached to a liquid-proof HDPE (high density polyethylene) membrane with a low Young's modulus to generate a pressure wave in the liquid reservoir. The experimental results showed that the atomizer can generate droplets of alcohol with a Sauter mean diameter (SMD) of $4.6 \mu \mathrm{m}$ at a flow rate of $2.5 \mathrm{~g} / \mathrm{min}$. The PZT-driven atomizer was tested under an operating voltage of $22 \mathrm{~V}$ and current of $180 \mathrm{~mA}$, the frequency was from 12 to $24 \mathrm{kHz}$, and the power needed to start up was $2 \mathrm{~W}$. During operation, a voltage was applied to the PZT bimorph plate causing the HDPE diaphragm to deflect continuously backward and forward. The volume change in the reservoir due to the diaphragm deflection in the forward direction caused an acoustic pressure wave to propagate towards the nozzles located at the front of the microatomizer 


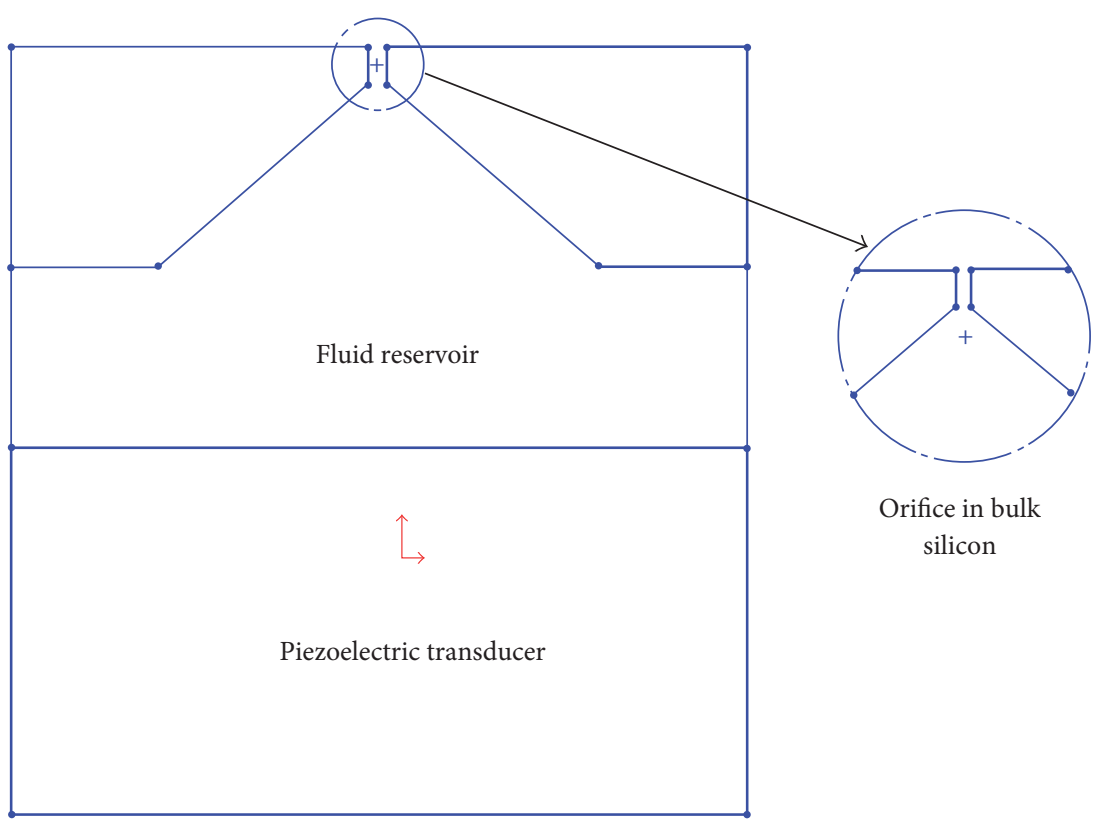

FIgURE 3: Schematic of a piezoelectrically driven, micromachined atomizer with passive mesh membrane.

expelling the droplets through the nozzle plate. Rearward deflection of the diaphragm drew liquid into the reservoir through the replenishment channel. By adjusting the driving waveform, voltage amplitude and frequency, different flow rates, and particle size distributions could be obtained.

\section{Discussion}

After going through various geometries and design methods, it can be easily understood that the geometry and the vibration mode play important roles in the ultrasound generation and power demands. Whereas uniform thickness plates are not capable of producing in-phase and focused waves, the stepped-thickness plates proved to be useful [22-24, 27]. Directivity patterns were compared in [23] for uniform and stepped-thickness plates and raising the steps to half a wave length of the radiated sound in the medium affected the directivity and strength of the ultrasound generation. However, using stepped-thickness plates for low frequencies is not practical due to the large height of the steps which in specific applications (i.e., small and thin transducers) may be a restriction specially for radiation in liquids and, therefore, not feasible. The operating frequency, size, and shape of the structure and the intended application clarify whether the steps are practically possible or not. Further, the acoustic medium is extremely influential to decide on the applicability of introducing steps in the thickness. Assuming water to be the acoustic medium with the sound velocity of approximately four times that of air, the thickness of the steps at the same frequency will be much higher than that of air and, as a consequence, it may not be viable to have steps. For many types of liquid medicine, the same problem exists. Going to higher frequencies in order to reduce the height of the steps may not be possible since higher frequencies have some barriers as overheating of the transducer as reported by Lozano et al. [51]. Therefore, alternatives should be sought to achieve highly directional and focused ultrasonic waves. One alternative can be implementing curved transducers which have shown to be good at generating strong ultrasound in air $[30,31]$. However, they have not been thoroughly investigated when in contact with liquids suiting them for drug delivery or atomization. Another alternative can be using cylindrical shells to increase the directivity and focus of the generated ultrasonic waves. García-Pérez et al. employed a shell vibrating at one of its resonance frequencies to produce a high level of focused acoustic intensity in the air inside the tube to dry the fruits [37]. They also noted that considering the air flow rate inside the chamber is an important factor affecting the acoustic field extensively. The high level of acoustic intensity achieved in this paper acknowledged the influence of geometry and vibration mode on the ultrasound generation. However, the power consumption of $75 \mathrm{~W}$ and electric drives needed may be suitable for industrial purposes, while they are not suitable for biomedical applications. According to this reference and their achievement, cylindrical shells seem a promising alternative to be scrutinized.

In conclusion, improving current devices for biomedical applications such as atomization, drug delivery, humidification, and lung therapy seems crucial considering the drawbacks of the current commercial devices as already mentioned. Broad droplet size distributions, low throughput, clogging of the mesh orifices for drug delivery equipment, and high power consumption and large size of the lung supportive devices should be eliminated. According to the literature and discussions made in this paper, the future investigations on such biomedical devices which could have industrial applications as well should be concentrated and targeted on the geometrical manipulation of the transducer 
TABLE 1: Summary of the available methods for atomization.

\begin{tabular}{|c|c|c|c|c|}
\hline Method & Schematic and properties & $\begin{array}{l}\text { Droplet size and } \\
\text { Flow rate }\end{array}$ & Advantages & Disadvantages \\
\hline SAW $[38,39]$ & $2.3 \mathrm{~W} ; 48 \mathrm{MHz}, 36 \mathrm{~V}$ & $\begin{array}{c}5 \mu \mathrm{m} \\
10.2 \mathrm{~mL} / \mathrm{h}\end{array}$ & Small size \& low power & $\begin{array}{c}\text { IDT fabrication process \& } \\
\text { very high frequency \& big } \\
\text { droplets }\end{array}$ \\
\hline $\begin{array}{l}\text { SAW driving structure consisting } \\
\text { of a unidirectional interdigital } \\
\text { transducer, a horn, and a } \\
\text { waveguide [40] }\end{array}$ & $1 \mathrm{~W} ; 78 \mathrm{MHz}$ & $\begin{array}{c}1.5 \mu \mathrm{m} \\
2.4 \mathrm{~mL} / \mathrm{h}\end{array}$ & $\begin{array}{l}\text { Small size \& smaller } \\
\text { droplets \& low power }\end{array}$ & $\begin{array}{l}\text { Very low flow rate \& IDT } \\
\text { fabrication \& high } \\
\text { frequency }\end{array}$ \\
\hline $\begin{array}{l}\text { Arrays of } 5 \mu \mathrm{m} \text { diameter } \\
\text { micromachined nozzles }[41,42]\end{array}$ & $36 \mathrm{kHz} ; 70,80 \mathrm{~V}$ & $\begin{array}{l}5-10 \mu \mathrm{m}, \\
\text { According to [19] } \\
\text { for typical } \\
\text { frequencies of } \\
\text { around } 100 \mathrm{kHz} \text {, it } \\
\text { is } 4 \mu \mathrm{m}\end{array}$ & Small size \& low frequency & Fabrication \& big droplets \\
\hline $\begin{array}{l}\text { MEM Fourier-horn ultrasonic } \\
\text { nozzle with central channel [43] }\end{array}$ & $0.25 \mathrm{~W} ; 971 \mathrm{kHz}$ & $\begin{array}{c}4.5 \mu \mathrm{m} \\
21 \mathrm{~mL} / \mathrm{h} \\
\end{array}$ & $\begin{array}{c}\text { Small size \& excellent low } \\
\text { power }\end{array}$ & $\begin{array}{c}\text { Fabrication \& big droplets } \\
\text { \& average flow rate }\end{array}$ \\
\hline $\begin{array}{l}\text { MEM Fourier-horn ultrasonic } \\
\text { nozzle without central channel } \\
\text { (externally fed) [17] }\end{array}$ & $80 \mathrm{~mW} ; 2 \mathrm{MHz}$ & $\begin{array}{l}2.9-4.6 \mu \mathrm{m} \\
25 \mathrm{~mL} / \mathrm{h}\end{array}$ & $\begin{array}{c}\text { Small size \& excellent low } \\
\text { power }\end{array}$ & $\begin{array}{c}\text { Fabrication \& average } \\
\text { droplets \& average flow rate }\end{array}$ \\
\hline $\begin{array}{l}\text { MEM Fourier-horn ultrasonic } \\
\text { nozzle without central channel } \\
\text { (externally fed) [18] }\end{array}$ & $0.27 \mathrm{~W} ; 2 \mathrm{MHz}$ & $\begin{array}{c}2-5 \mu \mathrm{m} \& \text { Typically } \\
=3.5 \mu \mathrm{m} \\
\text { Max } 21 \mathrm{~mL} / \mathrm{h} \& \\
\text { Typically }=9 \mathrm{~mL} / \mathrm{h}\end{array}$ & $\begin{array}{l}\text { Pocket Size \& battery } \\
\text { operated \& excellent low } \\
\text { power }\end{array}$ & $\begin{array}{c}\text { Fabrication \& average } \\
\text { droplets \& average or low } \\
\text { flow rate }\end{array}$ \\
\hline $\begin{array}{l}\text { Current vibrating plate } \\
\text { transducers [44-47] }\end{array}$ & $2-30 \mathrm{~W} ; 1.65-3 \mathrm{MHz}$ & $\begin{array}{c}1-5 \mu \mathrm{m} \\
5-400 \mathrm{~mL} / \mathrm{h}\end{array}$ & $\begin{array}{l}\text { Various droplet size and } \\
\text { flow rate }\end{array}$ & $\begin{array}{l}\text { Low-power devices with } \\
\text { small droplets have low } \\
\text { flow rate }\end{array}$ \\
\hline
\end{tabular}

to increase the acoustic intensity and its directivity which could in turn reduce the size of the transducer itself as well as corresponding electrical drives. The corresponding mode of vibration should also be carefully taken into account since it extensively affects the acoustic radiation. Therefore, extensive investigation on the proper mode of vibration for the considered geometry is essential. Such manipulation is anticipated to decrease the power demands since Gallego-Juárez et al. reported $147 \mathrm{~dB}$ of acoustic intensity with $350 \mathrm{~W}$ of power using five circular stepped-plate transducers covering a radiating area of about $1.64 \times 1.64 \mathrm{~m}^{2}$ [29]. Gallego-Juárez et al. managed to get approximately $140 \mathrm{~dB}$ with just $1 \mathrm{~W}$ and over $160 \mathrm{~dB}$ with $200 \mathrm{~W}$ (around $160 \mathrm{~dB}$ with $100 \mathrm{~W}$ ) for aluminum and titanium stepped plates of $200 \mathrm{~mm}$ diameter which were excited by means of a prestressed composite half-wavelength cylindrical transducer consisting of two piezoelectric ceramic rings [22]. García-Pérez et al. reported $154 \mathrm{~dB}$ with only $75 \mathrm{~W}$ employing an aluminum cylindrical shell of $100 \mathrm{~mm}$ diameter and $310 \mathrm{~mm}$ length which was driven by a piezoelectric composite transducer consisting of an extensional piezoelectric sandwich element together with a mechanical amplifier [37]. Although the size and/or power consumption of the abovementioned examples may not suit biomedical applications, the intensity and directivity of the generated ultrasonic field achieved by the geometrical manipulations can verify the great role of the geometry. Moreover, the focused transducer with high intensity may result in the elimination of mesh orifices in the new design approaches solving the blockage problem. All these should encourage the future investigations to be concentrated on the transducer geometry, its suitable vibration mode, and the strength and directivity of the generated acoustic field.

\section{Conclusion}

Due to a great deal of research on ultrasonic transducers and their widespread use in many different applications, they have been reviewed in this paper. Moreover, focus has been on reviewing the ultrasonic transducers as atomizers, which have a lot of biomedical and industrial applications. Various methods for generating focused ultrasound by means of flat plate transducers and shell configurations were presented. In the end, transducers for atomization were introduced. Table 1 summarizes the available approaches for atomization together with the positive and negative points of each method. As evident in the Table, vibrating plate atomizers possess the best combination of performing specifications, so that they are very common atomizers. None of the above methods with the exception of reference [37] produce complete ultrasound humidification such as that used for lung supportive devices. Biomedical humidification in lung therapies requires complete water vapor in the air rather than water droplets. For this humidification, it is preferable to have an in-line humidifier which can fit in a breathing tube of about $2 \mathrm{~cm}$ diameter such as those used in continuous positive airway pressure (CPAP) devices [59]. This paper can serve 
as a compact reference and guideline for various investigations on focused ultrasound generation as well as atomization using ultrasonic transducers.

\section{Disclosure}

This work was performed at the Institute of Biomedical Technologies (IBTec), Auckland University of Technology (AUT), Auckland, New Zealand.

\section{Competing Interests}

The authors declare that there is no conflict of interests in the submitted manuscript.

\section{References}

[1] K. Hettiarachchi, E. Talu, M. L. Longo, P. A. Dayton, and A. P. Lee, "On-chip generation of microbubbles as a practical technology for manufacturing contrast agents for ultrasonic imaging," Lab on a Chip, vol. 7, no. 4, pp. 463-468, 2007.

[2] J. S. Patton and P. R. Byron, "Inhaling medicines: delivering drugs to the body through the lungs," Nature Reviews Drug Discovery, vol. 6, no. 1, pp. 67-74, 2007.

[3] J. Heyder, "Deposition of inhaled particles in the human respiratory tract and consequences for regional targeting in respiratory drug delivery," Proceedings of the American Thoracic Society, vol. 1, no. 4, pp. 315-320, 2004.

[4] D. A. Edwards, J. Hanes, G. Caponetti et al., "Large porous particles for pulmonary drug delivery," Science, vol. 276, no. 5320, pp. 1868-1871, 1997.

[5] R. Langer, "Drugs on target," Science, vol. 293, no. 5527, pp. 5859, 2001.

[6] R. Langer and N. A. Peppas, "Advances in biomaterials, drug delivery, and bionanotechnology," AIChE Journal, vol. 49, no. 12, pp. 2990-3006, 2003.

[7] D. A. LaVan, T. McGuire, and R. Langer, "Small-scale systems for in vivo drug delivery," Nature Biotechnology, vol. 21, no. 10, pp. 1184-1191, 2003.

[8] M. N. Topp, "Ultrasonic atomization-a photographic study of the mechanism of disintegration," Journal of Aerosol Science, vol. 4, no. 1, pp. 17-25, 1973.

[9] B. Avvaru, M. N. Patil, P. R. Gogate, and A. B. Pandit, "Ultrasonic atomization: effect of liquid phase properties," Ultrasonics, vol. 44, no. 2, pp. 146-158, 2006.

[10] A. L. Yarin, D. A. Weiss, G. Brenn, and D. Rensink, "Acoustically levitated drops: drop oscillation and break-up driven by ultrasound modulation," International Journal of Multiphase Flow, vol. 28, no. 6, pp. 887-910, 2002.

[11] E. H. Trinh, "Compact acoustic levitation device for studies in fluid dynamics and material science in the laboratory and microgravity," Review of Scientific Instruments, vol. 56, no. 11, pp. 2059-2065, 1985.

[12] A. V. Anilkumar, C. P. Lee, and T. G. Wang, "Stability of an acoustically levitated and flattened drop: an experimental study," Physics of Fluids, vol. 5, no. 11, pp. 2763-2774, 1993.

[13] W. Tao Shi, R. E. Apfel, and R. Glynn Holt, "Instability of a deformed liquid drop in an acoustic field," Physics of Fluids, vol. 7, no. 11, pp. 2601-2607, 1995.
[14] A. L. Yarin, "Drop impact dynamics: splashing, spreading, receding, bouncing," Annual Review of Fluid Mechanics, vol. 38, pp. 159-192, 2006.

[15] M. Rein, "Phenomena of liquid drop impact on solid and liquid surfaces," Fluid Dynamics Research, vol. 12, no. 2, pp. 61-93, 1993.

[16] R. Rioboo, C. Tropea, and M. Marengo, "Outcomes from a drop impact on solid surfaces," Atomization and Sprays, vol. 11, no. 2, pp. 155-165, 2001.

[17] C. S. Tsai, R. W. Mao, S. K. Lin, N. Wang, and S. C. Tsai, "Miniaturized multiple fourier-horn ultrasonic droplet generators for biomedical applications," Lab on a Chip, vol. 10, no. 20, pp. 27332740, 2010.

[18] C. Tsai, R. Mao, S. Lin, Y. Zhu, and S. Tsai, "Faraday instabilitybased micro droplet ejection for inhalation drug delivery," Technology, vol. 2, no. 1, pp. 75-81, 2014.

[19] J. S. Lass, A. Sant, and M. Knoch, "New advances in aerosolised drug delivery: vibrating membrane nebuliser technology," Expert Opinion on Drug Delivery, vol. 3, no. 5, pp. 693-702, 2006.

[20] B. L. Rottier, C. J. P. van Erp, T. S. Sluyter, H. G. M. Heijerman, H. W. Frijlink, and A. H. de Boer, "Changes in performance of the pari eflow ${ }^{\circledR}$ rapid and pari LC plus ${ }^{\mathrm{TM}}$ during 6 months use by CF patients," Journal of Aerosol Medicine and Pulmonary Drug Delivery, vol. 22, no. 3, pp. 263-269, 2009.

[21] B. Mortimer, G. Saban, A. Samboer, and B. Verveckken, "The development of an ultrasonic humidifier for domestic applications," in Proceedings of the Domestic Use of Electrical Energy Conference, pp. 138-140, 1999.

[22] J. A. Gallego-Juárez, G. Rodriguez-Corral, and L. GaeteGarreton, "An ultrasonic transducer for high power applications in gases," Ultrasonics, vol. 16, no. 6, pp. 267-271, 1978.

[23] A. Barone and J. A. G. Juarez, "Flexural vibrating free-edge plates with stepped thicknesses for generating high directional ultrasonic radiation," The Journal of the Acoustical Society of America, vol. 51, no. 3B, pp. 953-959, 1972.

[24] J. A. Gallego Juárez, "Axisymmetric vibrations of circular plates with stepped thickness," Journal of Sound and Vibration, vol. 26, no. 3, pp. 411-416, 1973.

[25] J. L. San Emeterio, J. A. Gallego-Juárez, and G. RodriguezCorral, "IGH axisymmetric modes of vibration of stepped circular plates," Journal of Sound and Vibration, vol. 114, no. 3, pp. 495-505, 1987.

[26] A. M. Al-Jumaily and K. Jameel, "Influence of the Poisson ratio on the natural frequencies of stepped-thickness circular plate," Journal of Sound and Vibration, vol. 234, no. 5, pp. 881-894, 2000.

[27] F. Montero de Espinosa and J. A. Gallego-Juárez, "A directional single-element underwater acoustic projector," Ultrasonics, vol. 24, no. 2, pp. 100-104, 1986.

[28] J. A. Gallego-Juárez, G. Rodríguez-Corral, E. Riera-Franco de Sarabia, F. Vázquez-Martínez, V. M. Acosta-Aparicio, and C. Campos-Pozuelo, "Development of industrial models of highpower stepped-plate sonic and ultrasonic transducers for use in fluids," in Proceedings of the Ultrasonics Symposium, vol. 2, pp. 571-578, Atlanta, Ga, USA, October 2001.

[29] J. A. Gallego-Juárez, G. Rodríguez-Corral, E. Riera-Franco De Sarabia, F. Vázquez-Martínez, C. Campos-Pozuelo, and V. M. Acosta-Aparicio, "Recent developments in vibrating-plate macrosonic transducers," Ultrasonics, vol. 40, no. 1-8, pp. 889893, 2002. 
[30] H. Wang and M. Toda, "Curved PVDF airborne transducer," IEEE Transactions on Ultrasonics, Ferroelectrics, and Frequency Control, vol. 46, no. 6, pp. 1375-1386, 1999.

[31] M. Toda and S. Tosima, "Theory of curved, clamped, piezoelectric film, air-borne transducers," IEEE Transactions on Ultrasonics, Ferroelectrics, and Frequency Control, vol. 47, no. 6, pp. 1421-1431, 2000.

[32] M. Toda, "Phase-matched air ultrasonic transducers using corrugated PVDF film with half wavelength depth," IEEE Transactions on Ultrasonics, Ferroelectrics, and Frequency Control, vol. 48, no. 6, pp. 1568-1574, 2001.

[33] H. Du, L. Xu, H. Hu et al., "High-frequency vibrations of corrugated cylindrical piezoelectric shells," Acta Mechanica Solida Sinica, vol. 21, no. 6, pp. 564-572, 2008.

[34] L. Xu, M. Chen, H. Du et al., "Vibration characteristics of a corrugated cylindrical shell piezoelectric transducer," IEEE Transactions on Ultrasonics, Ferroelectrics, and Frequency Control, vol. 55, no. 11, pp. 2502-2508, 2008.

[35] H. Li, F. Yang, H. Du et al., "Dynamic characteristics of axiallysymmetrical annular corrugated shell piezoelectric transducers," Acta Mechanica Solida Sinica, vol. 22, no. 5, pp. 499-509, 2009.

[36] F. Blum, J. Jarzynski, and L. J. Jacobs, "A focused two-dimensional air-coupled ultrasonic array for non-contact generation," NDT and E International, vol. 38, no. 8, pp. 634-642, 2005.

[37] J. V. García-Pérez, J. A. Cárcel, S. de la Fuente-Blanco, and E. Riera-Franco de Sarabia, "Ultrasonic drying of foodstuff in a fluidized bed: parametric study," Ultrasonics, vol. 44, pp. e539e543, 2006.

[38] M. Kurosawa, T. Watanabe, A. Futami, and T. Higuchi, "Surface acoustic wave atomizer," Sensors and Actuators: A. Physical, vol. 50, no. 1-2, pp. 69-74, 1995.

[39] M. Kurosawa, A. Futami, and T. Higuchi, "Characteristics of liquids atomization using surface acoustic wave," in Proceedings of the International Conference on Solid State Sensors and Actuators (TRANSDUCERS '97), IEEE, Chicago, Ill, USA, June 1997.

[40] W. Soluch and T. Wrobel, "Low driving power SAW atomiser," Electronics Letters, vol. 42, no. 24, Article ID 1432, 2006.

[41] S. Yuan, Z. Zhou, and G. Wang, "Experimental research on piezoelectric array microjet," Sensors and Actuators A: Physical, vol. 108, no. 1-3, pp. 182-186, 2003.

[42] S. Yuan, Z. Zhou, G. Wang, and C. Liu, "MEMS-based piezoelectric array microjet," Microelectronic Engineering, vol. 66, no. 1-4, pp. 767-772, 2003.

[43] S. C. Tsai, C. H. Cheng, N. Wang, Y. L. Song, C. T. Lee, and C. S. Tsai, "Silicon-based megahertz ultrasonic nozzles for production of monodisperse micrometer-sized droplets," IEEE Transactions on Ultrasonics, Ferroelectrics, and Frequency Control, vol. 56, no. 9, pp. 1968-1979, 2009.

[44] L. APC International, Ed., $1.65 \mathrm{mhz}$ Nebulizer Boards Catalog, 2014.

[45] Siansonic general atomizing kit catalog, S.T.C. Ltd.

[46] Siansonic mini nebulizer kit catalog, S.T.C. Ltd.

[47] Siansonic high efficiency atomizer transducer catalog, S.T.C. Ltd.

[48] S. Sangwan, R. Condos, and G. C. Smaldone, "Lung deposition and respirable mass during wet nebulization," Journal of Aerosol Medicine: Deposition, Clearance, and Effects in the Lung, vol. 16, no. 4, pp. 379-386, 2003.
[49] T. G. O’Riordan, L. I. Kleinman, K. Hughes, and G. C. Smaldone, "Predicting aerosol deposition during neonatal ventilation: feasibility of bench testing," Respiratory Care, vol. 39, no. 12, pp. 1162-1168, 1994.

[50] S. A. Elrod, B. Hadimioglu, B. T. Khuri-Yakub, E. G. Rawson, and C. F. Quate, "Focused acoustic beams for nozzleless droplet formation," in Proceedings of the Ultrasonics Symposium, IEEE, Chicago, Ill, USA, 1988.

[51] A. Lozano, H. Amaveda, F. Barreras, X. Jordà, and M. Lozano, "High-frequency ultrasonic atomization with pulsed excitation," Journal of Fluids Engineering, Transactions of the ASME, vol. 125, no. 6, pp. 941-945, 2003.

[52] J. Ju, Y. Yamagata, H. Ohmori, and T. Higuchi, "High-frequency surface acoustic wave atomizer," Sensors and Actuators, A: Physical, vol. 145-146, no. 1-2, pp. 437-441, 2008.

[53] A. Lal and R. M. White, "Micromachined silicon ultrasonic atomizer," in Proceedings of the 1996 IEEE Ultrasonics Symposium, pp. 339-342, IEEE, San Antonio, Tex, USA, November 1996.

[54] T. Li, Y. Chen, and J. Ma, "Development of a miniaturized piezoelectric ultrasonic transducer," IEEE Transactions on Ultrasonics, Ferroelectrics, and Frequency Control, vol. 56, no. 3, pp. 649-659, 2009.

[55] S. C. Tsai, Y. L. Song, T. K. Tseng, Y. F. Chou, W. J. Chen, and C. S. Tsai, "High-frequency, silicon-based ultrasonic nozzles using multiple fourier horns," IEEE Transactions on Ultrasonics, Ferroelectrics, and Frequency Control, vol. 51, no. 3, pp. 277-285, 2004.

[56] C. S. Tsai, R. W. Mao, S. K. Lin, E. Chien, and S. C. Tsai, "MEMS-based multiple fourier-horn silicon ultrasonic atomizer for inhalation drug delivery," in Proceedings of the IEEE International Ultrasonics Symposium (IUS '11), pp. 1119-1122, Orlando, Fla, USA, October 2011.

[57] J. M. Meacham, A Micromachined Ultrasonic Droplet Generator: Design, Fabrication, Visualization, and Modeling, School of Mechanical Engineering, Georgia Institute of Technology, 2006.

[58] Y.-R. Jeng, C.-C. Su, G.-H. Feng, Y.-Y. Peng, and G.-P. Chien, "A PZT-driven atomizer based on a vibrating flexible membrane and a micro-machined trumpet-shaped nozzle array," Microsystem Technologies, vol. 15, no. 6, pp. 865-873, 2009.

[59] A. M. Al-Jumaily and P. Reddy, Medical Devices for Respiratory Dysfunction: Principles and Modeling of Continuous Airway Pressure (CPAP), ASME, New York, NY, USA, 2012. 


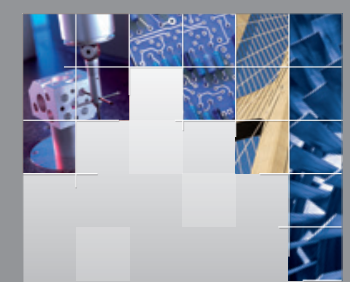

\section{Enfincering}
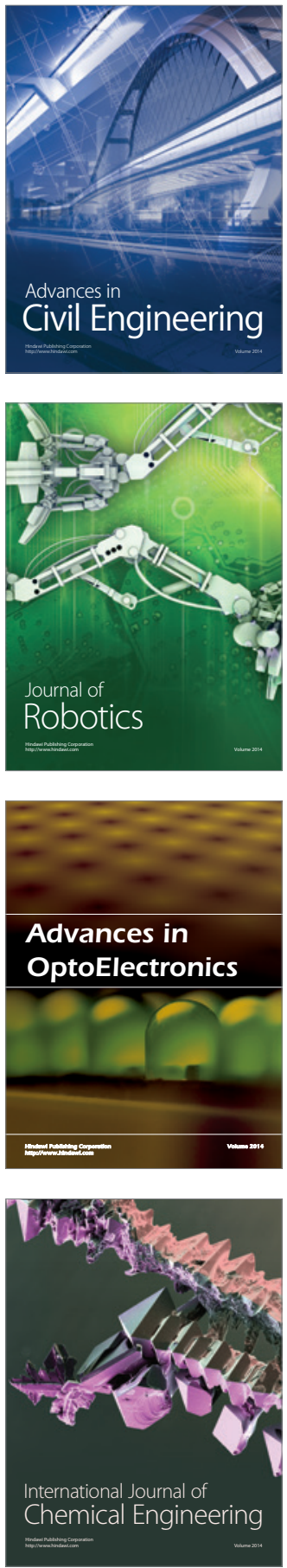

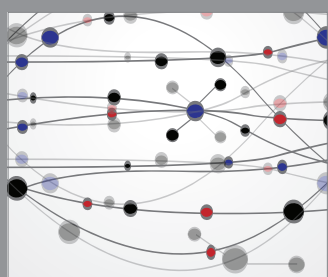

The Scientific World Journal

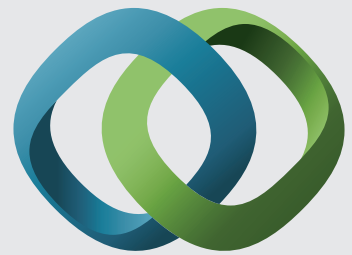

\section{Hindawi}

Submit your manuscripts at

https://www.hindawi.com
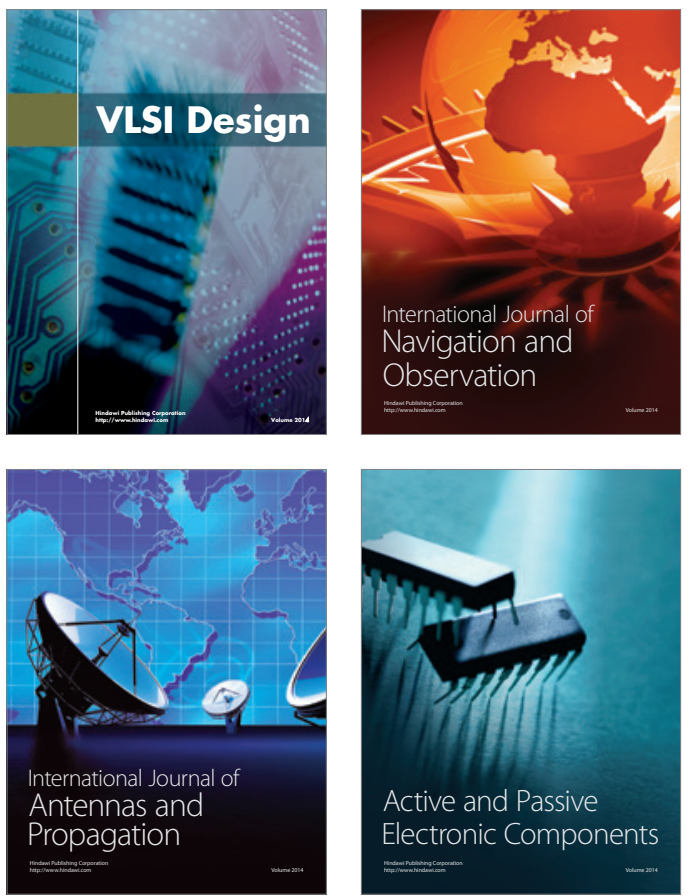
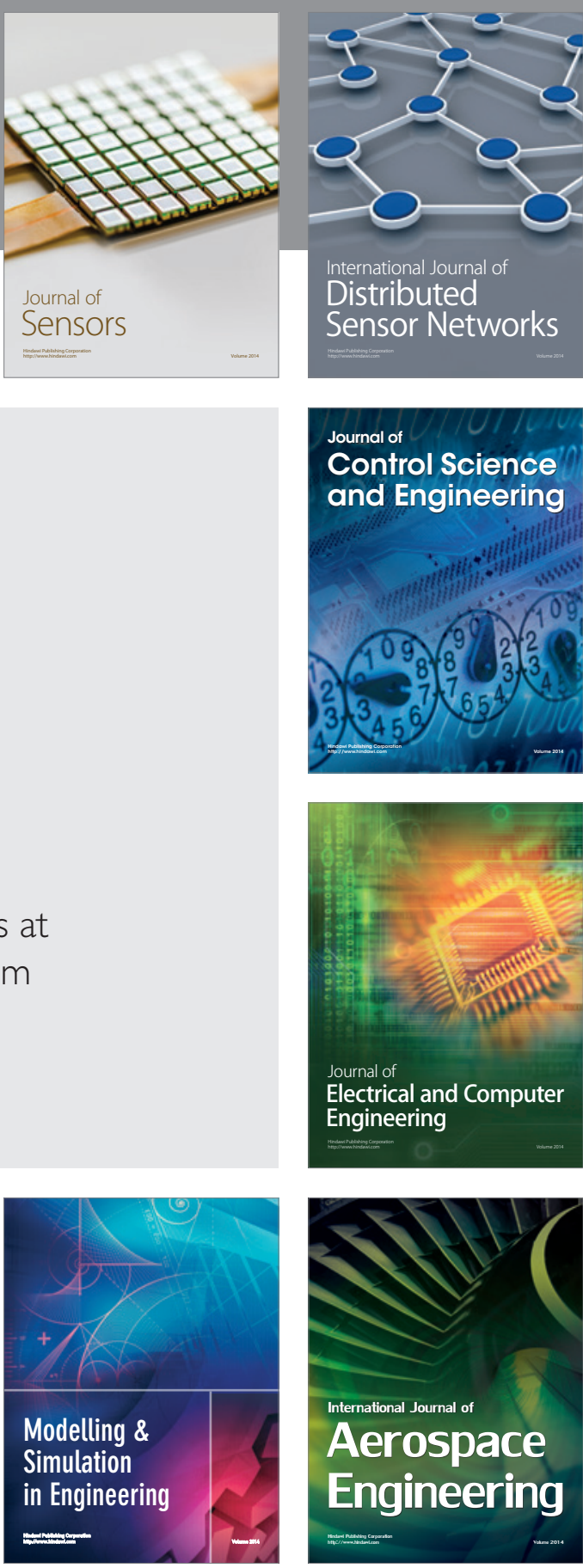

International Journal of

Distributed

Sensor Networks

$-$

Joumal of

Control Science

and Engineering
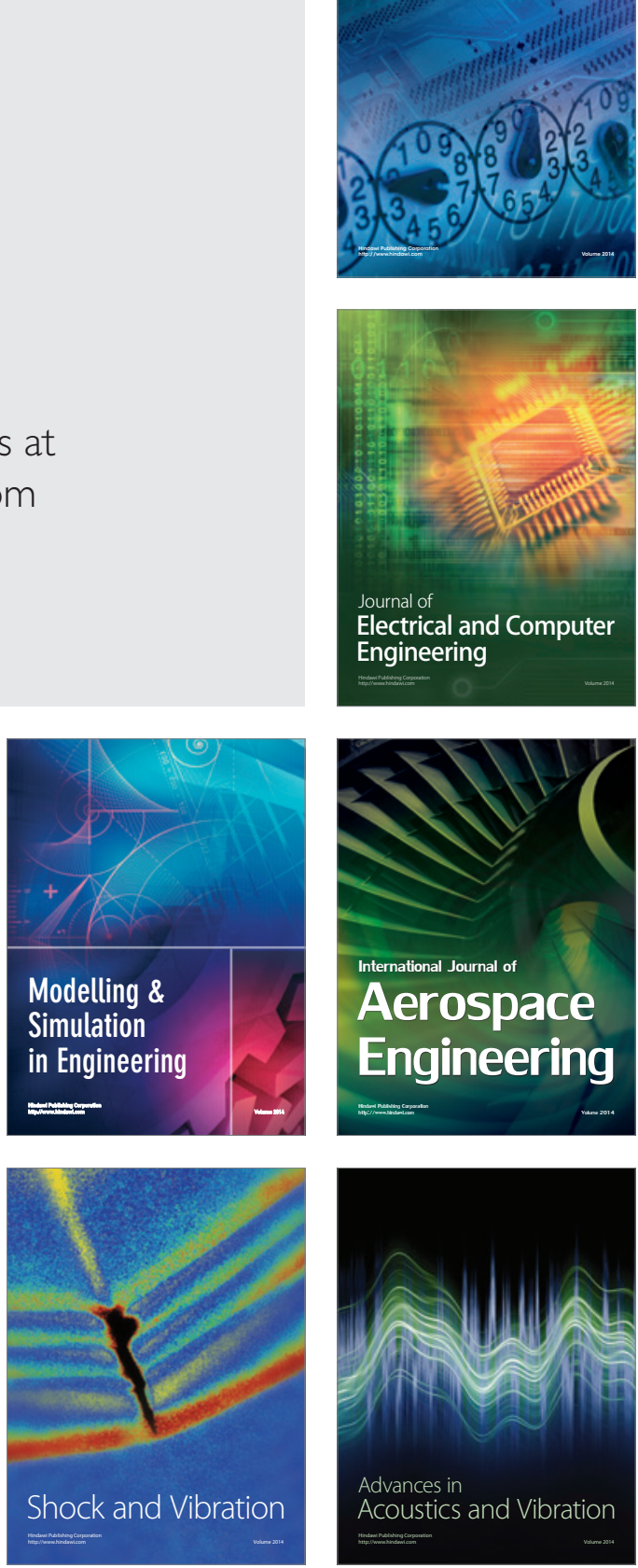